\title{
Research on Defect Image Enhancement in Ultrasonic TOFD Detection
}

\author{
$\mathrm{Li} \mathrm{Li}^{1, *}$, Kai Jiang ${ }^{1}$ and Fangqin $\mathrm{Li}^{2}$
}

${ }^{1}$ Hubei Key Laboratory of Hydroelectric Machinery Design \& Maintenance Three Gorges University, Yichang 443002, Hubei, China; ${ }^{2}$ Department of Electrical and Computer Engineering (ECE), National University of Singapore, 21 Lower Kent Ridge Road, Singapore 119077

\begin{abstract}
TOFD), the image signal detected is often reshaped and resulted in inaccuracy of defect measurements because the probe beam has a limited range. This paper proposed an image enhancement method based on synthetic aperture focusing technique (SAFT). A mathematical model of testing image was first established based on the generation process of the image. A method was then designed for detecting defects in near surface blind area based on filtering of through waves. Finally, a synthetic aperture focusing algorithm based on peak capture pretreatment was used to enhance the resolution of the image. By applying the above method to test a crack defect of $15 \mathrm{~mm}$, the method was able to accurately judge for crack defect. Also, the measuring accuracy can be improved by $6.4 \%$ compared to that of traditional detection method.
\end{abstract}

Keywords: Defect, image enhancement, near surface blind area, synthetic aperture focusing technique (SAFT), time of flight diffraction (TOFD).

\section{INTRODUCTION}

Ultrasonic time of flight diffraction (TOFD) is an ultrasonic testing method which can detect defects by analyzing the diffraction signals from the boundary of defects. It has a high rate of defect detection and is able to produce real-time imaging, so that the method is widely used in the detection of welding seam of steel structures [1]. During the actual detection, two probes with ultrasonic sensors are placed and moved in the direction perpendicular to the welding. At every detection point, A-scanning signals are collected. All the A-scanning signals are digitized and arranged to synthesize a digital processed image called B-scanning image. Due to the existence of surface through waves on B-scanning images and the certain width of the through waves, the diffraction signals near the surface defects are likely to be covered by the through waves, so that the defect detection characteristics are not obvious. Therefore, a blind area with a certain depth can be formed on the surface of TOFD method is used [2]. Meanwhile, the blind area will lead to the inaccuracy of detections, or even miss detections.

Generally, TOFD detection method uses probes with small size and big diffusion angle to expand the covering range by acoustic effect within the detected device. This is good for capturing diffraction signals and reducing the miss detection rate. However, the diffusion property of sound beam will produce defected $\mathrm{A}$-scanning signals with large redundant information, and cause defected B-scanning images to have a parabola shape, so that the resolution will be reduced in the horizontal direction [3]. Additionally, attenuation oscillation will be produced by sound waves, and the change in values makes it difficult to acquire accurate results in the vertical direction. Hence, the longitudinal resolution of the images will also be reduced. These reduces in resolution will lead to errors when using images to locate defects.

From the research of using ultrasonic TOFD to detect defects in near surface blind area, literature [4] has proposed to use pulse reflection method to solve the blind area problem in ultrasonic TOFD on the surface. It suggests using a straight probe with longitudinal waves to sweep through all regions, so that the defects near surface blind area can be detected. This method is theoretically feasible, but is only limited for detecting defects on smooth surfaces. As stated by pulse reflection method, the detecting probe must directly in contact with the surface of detected object. In other words, this method is not feasible when the welding surface is rough or uneven. Furthermore, literatures $[5,6]$ have proposed to acquire near surface defects signals by using longitudinal waves to reflect three times because the reflected waves will hit the near surface blind area. The spread time difference between longitudinal waves and surface through waves is huge, so that they are easy to be distinguished from detected images. However, this method can effectively detect artificial defects with depth of $1 \mathrm{~mm}$ as it has limitations, for example, the signals received are too complex and are not easy to be analyzed.

To improve the image resolution, image enhancement method is generally used, which includes: sparse deconvolution method [7, 8], dynamic frequency scanning [9] and synthetic aperture focusing technique (SAFT) [10]. Deconvolution method can eliminate the effect of testing probes, coupling, conditions and effects of noises. It can also highlight signals which contain the characteristics of the device under 


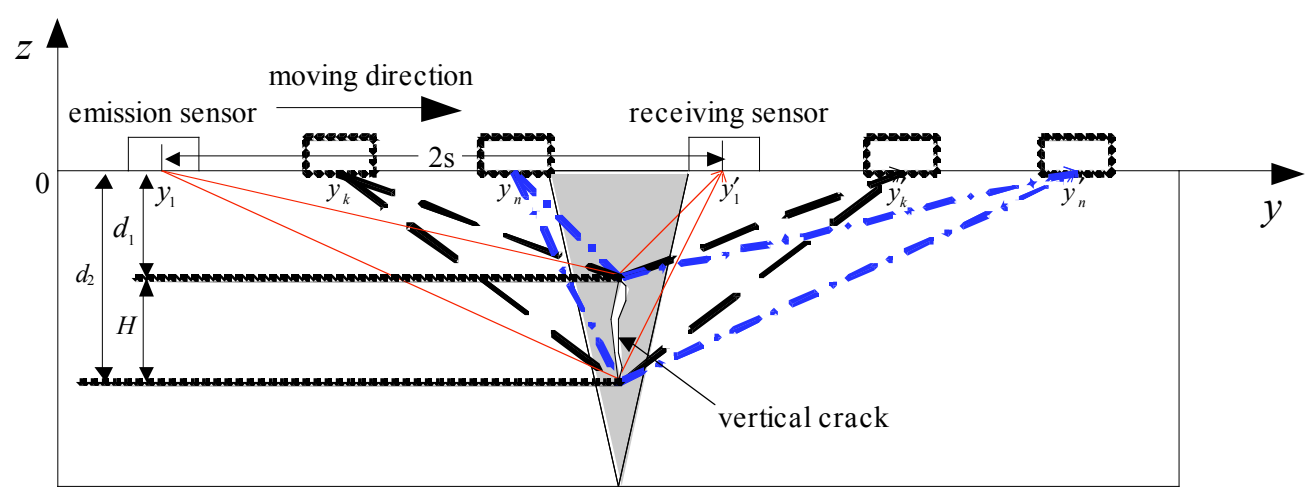

Fig. (1). Cross-section of the welded steel plate.

detection to improve the time resolution. However, the beam of ultrasonic TOFD probes contains a variety of frequencies. These ultrasonic testing signals of different frequency components have a significant influence on signal detection, which makes it difficult to select reference waveforms when using sparse deconvolution method. In other words, regardless of the choice of reference signals, it is difficult to achieve good results. Dynamical multi-frequency scanning technology is made up of a probe with a fixed frequency instead of multiple probes of different frequencies. Hence, the image will not degrade due to attenuation and can substantially improve the image resolution. However, the probe used has special requirements which are difficult to achieve. Synthesis aperture focused technology (SAFT) was first used for synthesis aperture radar technology to improve the horizontal resolution of radar images, literatures [10-12] apply SAFT application in ultrasonic TOFD imaging, and have achieved good research results. However, the limitation of this research is that the longitudinal resolution of SAFT imaging is still low, especially for near surface defects on devices. The through waves and diffraction waves are easily overlap with each other, preventing the process of SAFT imaging.

In order to solve the problems of blind areas and the inaccuracy in defects allocation when using ultrasonic TOFD on near surface, this paper has constructed a mathematical model based on the process of generating B-scanning images. By using this model to straighten and filter the through waves based on energy distribution law, this paper has solved the problem to detect defects on the near surface by using ultrasonic TOFD method. Meanwhile, this paper has presented an algorithm of synthetic aperture focusing based on pre-processing of peak values captured. By analyzing a case study of welding defects, the algorithm is able to largely improve the resolution of B-scanning images and the measurement accuracy of cracks and defect signals by using ultrasonic TOFD image detection.

\section{PRINCIPLE OF SAFT IMAGE ENHANCING METHOD}

\subsection{Method of Ultrasonic TOFD Testing Image Generation}

The obtained B-scanning images from ultrasonic TOFD method are built in sequence by a series arrangement of Ascanning signals [4]. It can be illustrated by using an exam- ple of a welded steel plate with a vertical crack placed inside, and the cross-section of the plate is as shown in Fig. (1). The example takes two probes placed on both sides of the weld and performs scanning in the direction perpendicular to the weld. In other words, the transmitting and receiving probes are moved from position $y_{1}-y_{1}^{\prime}$ to position $y_{n}-y_{n}^{\prime}$ via position $y_{k}-y_{k}^{\prime}$. When the probe is in position $y_{k}-y_{k}^{\prime}$, the crack defects are on the perpendicular bisector of the connection line of two probes. The A-scanning signal is generated when the probe moves a step distance of $\ddot{A} S$. After collecting all the A-scanning signals, a digitizing process is performed. First, the heights of the scanning signal waves at various points are expressed using numbers between $0-225$, then, all the numbers are normalized and expressed in digital grayscale values. For example, when the A-scanning signal at certain point reaches a positive full screen height, that is, when the number corresponds to 1 , the grayscale value of 225 is taken, and is represented in black; when A-scanning signal at certain point reaches a negative full screen height, that is, when the number corresponds to 0 , the grayscale value is 0 and is represented in white; when the wave height of the A-scanning signal at certain point is 0 , which corresponds to 0.5 , the grayscale value is taken as 128 and is represented in gray; the A-scanning signals at other points on the waveform are treated in the same manner. By using the above method to process all the A-scanning signals, and sequentially arrange all the digitized A-scanning signals, the B-scanning image can be generated. The image forming process is shown in Fig. (2).

According to the geometry relationship between defects and the probe, it can be shown that the arrival time of the defect diffraction wave signal at $y_{k}-y_{k}^{\prime}$ is earlier than that of A-scanning signals on both sides. The determination of defects using scanning images is based on the assumption that the defects are located on the perpendicular bisector of the connection line of two. Only under this assumption, the defect diffraction wave signal is able to provide accurate information of crack position and quantization. However, the original B-scanning image contains clutter of information, and the image resolution is very low, which results in large errors in determination of crack defects [13]. 


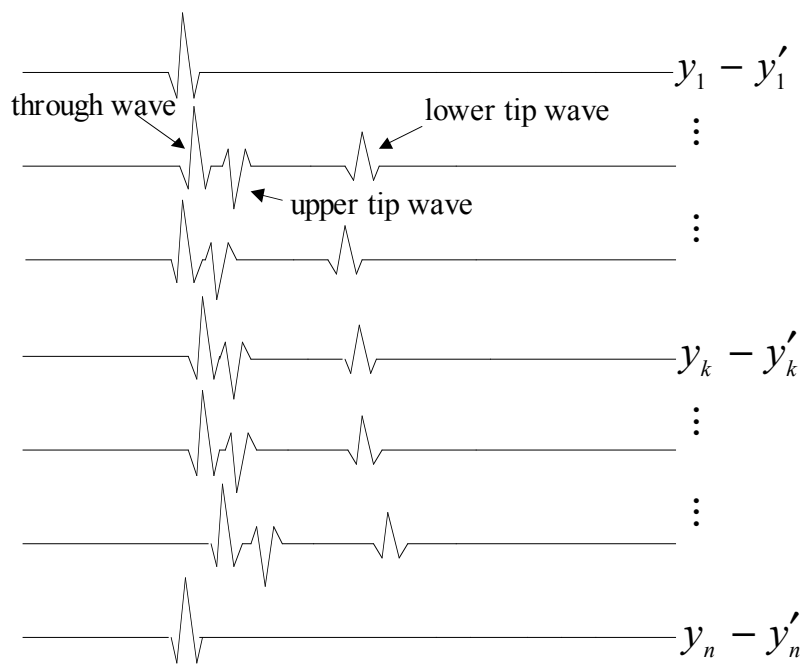

(a). wave diagram

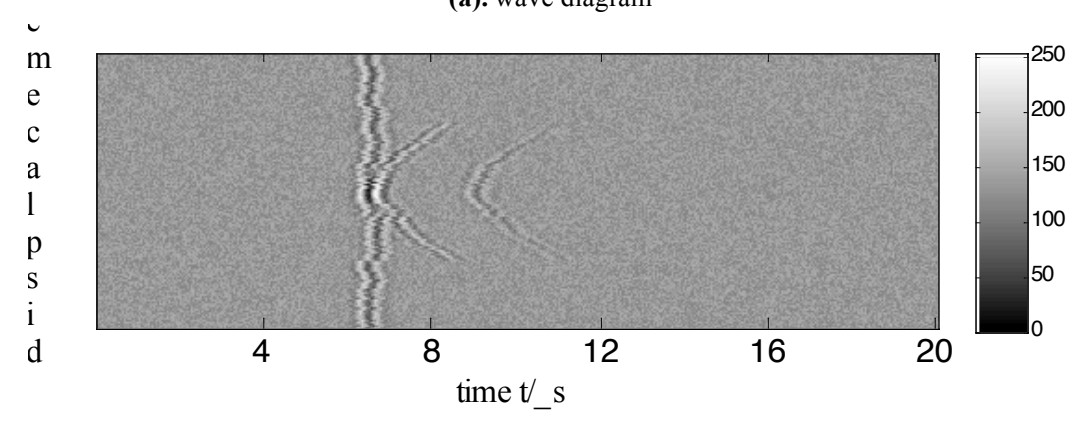

(b). B-scanning image

Fig. (2). Process of generating B-scanning image.

For the welding device, it is assumed that the distance between the probe and the center is $2 s$. The distance from lower and upper boundaries of defects to the surface of the device are $d_{1}$ and $d_{2}$ respectively. The vertical projection of the defect is $H$ as shown in Fig. (1). According to above geometric relationship, a formula can be generated as:

$\mathrm{H}=\mathrm{d}_{2}-\mathrm{d}_{1}=\sqrt{\mathrm{t}_{2}^{2} \mathrm{c}^{2}+\frac{1}{4} \mathrm{t}^{2} \mathrm{c}^{2}}-\sqrt{\mathrm{t}_{1}^{2} \mathrm{c}^{2}-\frac{1}{4} \mathrm{t}^{2} \mathrm{c}^{2}}$

$c$ is the longitudinal wave velocity in steel; $t_{1}$ and $t_{2}$ are the corresponding diffraction wave propagation times from lower and upper boundaries of the crack, and $t$ is the through wave propagation time.

From the composition and generation process of detected images, the ultrasonic TOFD detection signal can be expressed as [14]:

$f_{i j}(t)=x_{i j}(t)+d_{i j}(t)+c_{i j}(t), i=1 \sim M, j=1 \sim N$

In the formula, $\mathrm{x}_{\mathrm{ij}}(\mathrm{t})$ is the diffraction signal between upper and lower boundaries of the defect; $d_{i j}(t)$ is the through wave signal; $\mathrm{c}_{\mathrm{ij}}(\mathrm{t})$ is the noise signal; $i$ represents that B-scanning image is formed by arranging $\mathrm{M}$ A-scanning signals; $j$ means that for every A-scanning signal there are
$\mathrm{N}$ time points. Therefore, B-scanning image can be represented by $M \times N$ points.

\subsection{Method SAFT Pre-Processing Method}

In the ultrasonic TOFD B-scanning image, the certain width of the through wave will make it impossible to identify the diffraction signals from the boundary points of defect near the surface. The detected signals may be submerged in through wave signals to form a surface blind area [2], which is not allowed in the actual testing. Also, through wave signals are not used as detection signals and they will introduce redundant data in the process of enhancing detected signals. Therefore, in order to use SAFT, straightening, filtering and other processing methods should be used to process through waves beforehand.

\subsubsection{Through Wave Straightening Process}

During ultrasonic TOFD, the detection probes are in direct contact with the device surface. In the cases where there is changing coupling status or roughness on the device surface, inconsistency of through wave arrival times will be introduced in detection images, resulting in disordered images as shown in Fig. (2). From the figure, the diffraction wave of defect points at upper boundary links together with the through wave, this is because the upper boundary point is located near the surface blind area of the defect, so that it is unable to accurately read the upper boundary point diffraction wave information. Therefore, it is necessary to filter the 
through waves. In addition, some disorder trends can be observed in the beginning when the through waves appear, so that the waves need to be straightened before filtering.

As the propagation distance of the through wave is the shortest, it is the first to reach the receiver probe, and with the minimum energy loss and maximum amplitude. Thus, through wave straightening process can be carried out on the image. First, each A-scanning signal is normalized, and the timing points $t_{1} \cdots t_{i} \cdots t_{M}$ corresponding to each signal with value 1 are obtained respectively. The minimum value of timing point is assigned to be $t$. When $t_{i}-t>0$, the $i$ th signal is shifted ahead by $t_{i}-t$ units. Finally, the processed A-scanning signals are synthesized together, and the reconstructed image is as shown in Fig. (3). In the figure, through waves of every A-scanning signal are located on the same timing point, this is to facilitate the subsequent elimination of the through waves in the TOFD image.

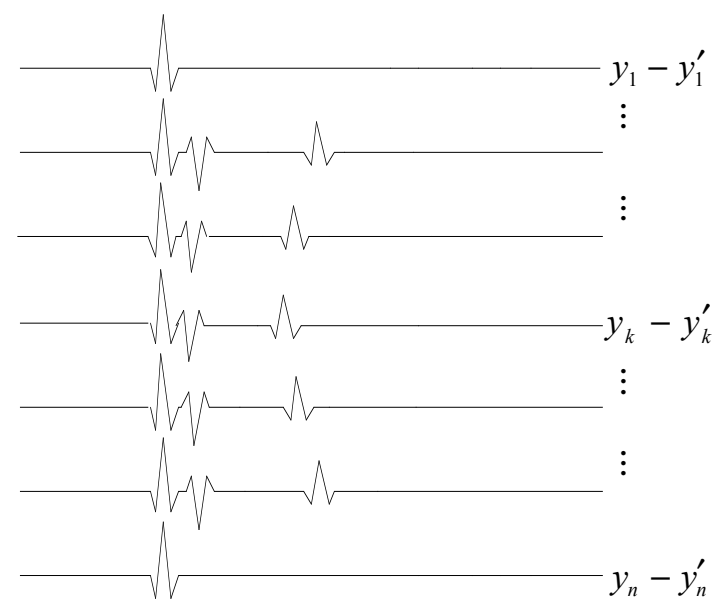

Fig. (3). Graph of through wave after straightening process.

\subsubsection{Through Wave Filtering Process}

Fig. (3) shows that in B-scanning images, the vibration of A-scanning signals containing defect waves is stronger than that of signals containing only through waves. Thus, extracting and filtering through waves can be done by image energy distribution method.

For B-scanning images to be processed, the energy of the $i$ th A-scanning signal can be expressed as [15]:

$E_{i}=\sum_{j=1}^{N} f(i, j)^{2}$

The energy of $\mathrm{M}$ number of A-scanning signals are obtained and normalized

$$
E_{i}^{\prime}=\frac{E_{i}}{\max \left(E_{1}, \cdots, E_{i}, \cdots, E_{M}\right)}
$$

Meanwhile, threshold value $T$ can be set, and Ascanning signals containing only through waves are defined with energy smaller than $T$. Assume there are $k$ such A- scanning signals, the average value of A-scanning signals containing only through waves can be expressed as:

$d_{j}=\left(f_{1 j}^{\prime}+\cdots+f_{k j}^{\prime}\right) / k$

In the B-scanning image, all A-scanning signals are subtracted by $d_{j}$, and the image is reconstructed so that image B contains only the defect signals as shown in Fig. (4a). As such, the through waves can be filtered out, and the diffraction signals inside the through waves can be identified.

\section{IMAGE ENHANCING METHOD BASED ON SAFT ULTRASONIC TOFD}

\subsection{Synthetic Aperture Focusing Mathematical Model Algorithm}

When the probe is positioned at $y_{k}-y_{k}^{\prime}$, the acquired A-scanning signal is $x_{n}$, and the propagation time of its corresponding diffraction wave on the crack boundary point is:

$t=2 \sqrt{s^{2}+d^{2}} / c$

In this formula, $S$ is half of the surface through wave propagation distance, $d$ is the vertical distance from the upper boundary of the crack to the surface, and $c$ is the longitudinal wave velocity in the material.

Assuming the step length is $\Delta s$, the propagation time of corresponding diffraction wave $x_{n+1}$ from position $y_{k}-y_{k}^{\prime}$ to the next position with step length $\Delta s$ is expressed as:

$t_{\Delta s}=\left(\sqrt{(s-\Delta s)^{2}+d^{2}}+\sqrt{(s+\Delta s)^{2}+d^{2}}\right) / c$

The delay time is:

$\ddot{A} t_{\ddot{A s}}=t_{\ddot{A} s}-t=\left(\sqrt{(s-\ddot{A} s)^{2}+d^{2}}+\sqrt{(s+\ddot{A} s)^{2}+d^{2}}-2 \sqrt{s^{2}+d^{2}}\right) / c$

Similarly, the propagation time of corresponding diffraction wave $x_{n+k}$ from position $y_{k}-y_{k}^{\prime}$ to the next position after k steps with step length $\Delta s$ is expressed as:

$t_{k \Delta s}=\left(\sqrt{(s-k \Delta s)^{2}+d^{2}}+\sqrt{(s+k \Delta s)^{2}+d^{2}}\right) / c$

The delay time is:

$\ddot{A} t_{k \ddot{A} s}=t_{\ddot{A} s}-t=\left(\sqrt{(s-k \ddot{A} \mathrm{~s})^{2}+d^{2}}+\sqrt{(s+k \ddot{A} \mathrm{~s})^{2}+d^{2}}-2 \sqrt{s^{2}+d^{2}}\right) / c$

The image enhancement process mainly includes signal shifting, arithmetic summation and averaging. During shifting operation, the chosen A-scanning signals are shifted accordingly based on the above calculation of delay times. In order to reduce the amount of computation to improve the processing speed, window processing method is used while 
the window width is the number of A-scanning signals participating in the shifting operation.

Also, the A-scanning signals in pre-processing region of B-scanning images need to undergo synthesis aperture focusing processing. The process is elaborated below and the reconstructed B-scanning images are shown in Fig. (4b).

$x_{n}\left(t_{n}\right)=1 /(N+1) \sum_{i=n-N / 2}^{n+N / 2} x_{i}\left(t_{i}-\Delta t_{i}\right)$

$x_{n}\left(t_{n}\right)$ is the returning signal of SAFT processed Ascanning signal $x_{n}$ at diffraction wave arrival time $t_{n} ; \mathrm{N}$ is the number of signals involved in SAFT operation when computing A-scanning signals on both sides of $x_{n}$, the operation window width is $N+1 ; x_{i}$ is the $i$ th A-scanning signal after shifting operation; $\Delta t_{i}$ is the time shifted.

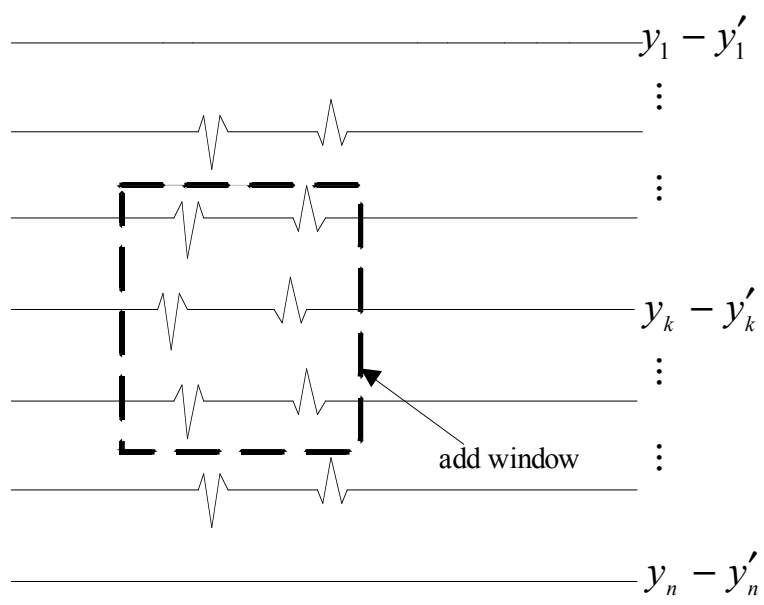

(a). Graph of through waves after filtering

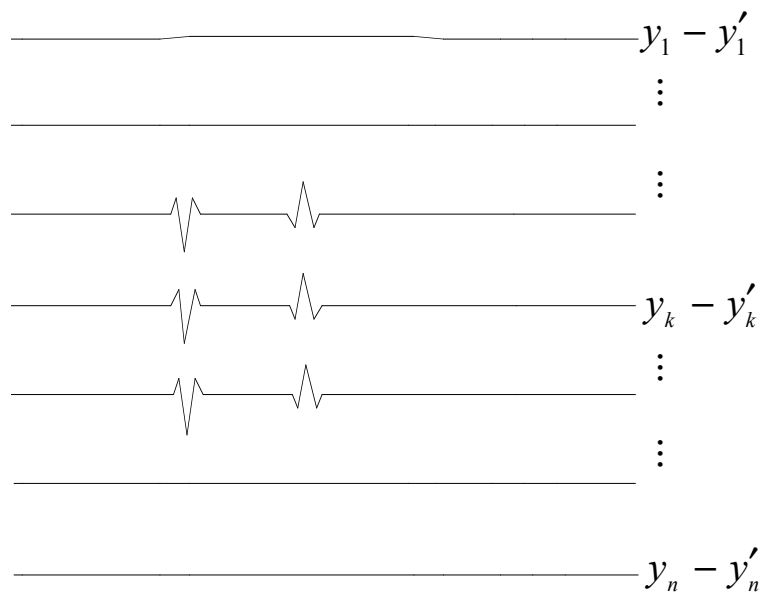

(b). Graph of through waves after synthesis aperture focusing process

Fig. (4). B-scanning image after synthesis aperture focusing process.

\subsection{Peak Value Capturing and Processing}

In order to improve the vertical resolution of the image, i.e. temporal resolution, peak values have been captured in B-scanning images after through waves filtering, with refer- ence to Fig. (4a). The grayscale values of digitized Ascanning signals are extracted, and the maximum values are retained while the other grayscale values are all set to be 128. The process is as shown in Fig. (5). Peak value capturing process can eliminate redundant signals such as attenuation of the acoustic oscillations, and can also confirm the defect diffraction wave arrival time of A-scanning signals.

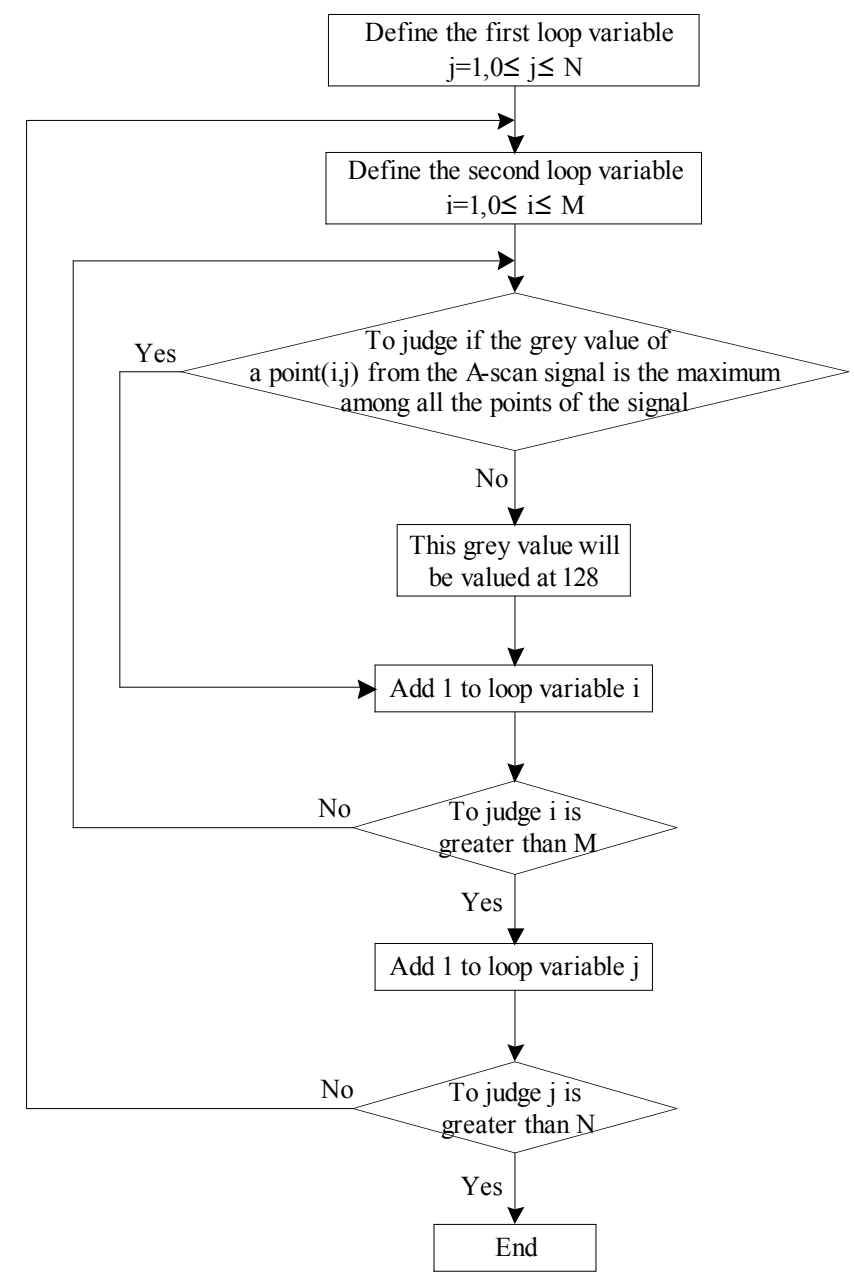

Fig. (5). Flow graph of peak value capturing process.

\section{CASE STUDY}

Ultrasonic TOFD method is applied to a welded steel plate with length of $150 \mathrm{~mm}$ and thickness of $45 \mathrm{~mm}$. A vertical crack is placed inside the plate with a length of $15 \mathrm{~mm}$. The distance from upper boundary point to surface of the plate is $1.5 \mathrm{~mm}$, and that from lower boundary point to surface of the plate is $16.5 \mathrm{~mm}$. Two round angle beam probes are used for transmitting and receiving signals. The probe centre operating frequency is $5 \mathrm{MHz}$, the wafer diameter is $10 \mathrm{~mm}$, the centre distance of the probe is $75 \mathrm{~mm}$, and the scanning step is $1 \mathrm{~mm}$. Fig. (6) shows the original B-scanning image of steel plate by using ultrasonic TOFD method, the image size is $200 \times 1000$. From the diagram, diffraction signals on upper boundary point of the defect are buried in the through waves, and a high degree of disorder can be observed from both defect waves and through waves. Also, the horizontal and vertical resolutions of the image are very 
poor, so that it is unable to precisely position and quantize the crack.

According to the B-scanning image shown in Fig. (6), the image first undergoes straightening and reconstructing processes. The normalized A-scanning signal located at position $y_{k}-y_{k}^{\prime}$ is shown in Fig. (7), and the corresponding time point when its amplitude equals to 1 is $6.46 \mu s$. The mini- mum corresponding time point when the amplitude equals to 1 in all A-scanning signals is $6.28 \mu \mathrm{s}$, and the A-scanning signal at position $y_{k}-y_{k}^{\prime}$ is shifted ahead by $0.18 \mu \mathrm{s}$. Similarly, every A-scanning signal undergoes the above shifting process to give a B-scanning image after straightening and reconstructing processes as shown in Fig. (8). 


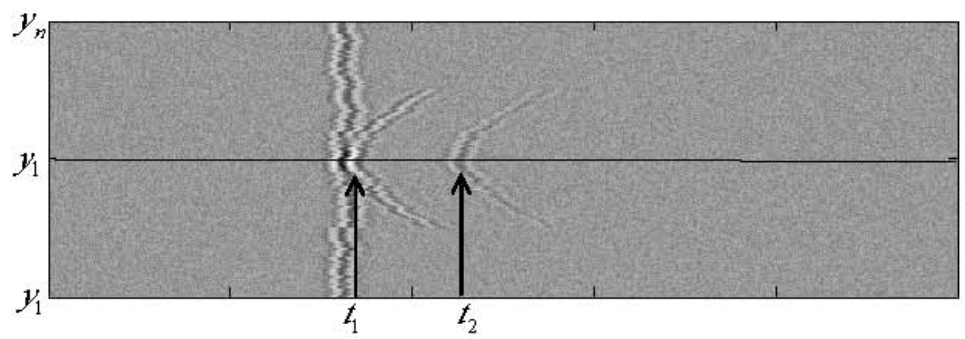

Fig. (6). Actual B-scanning image after detection.

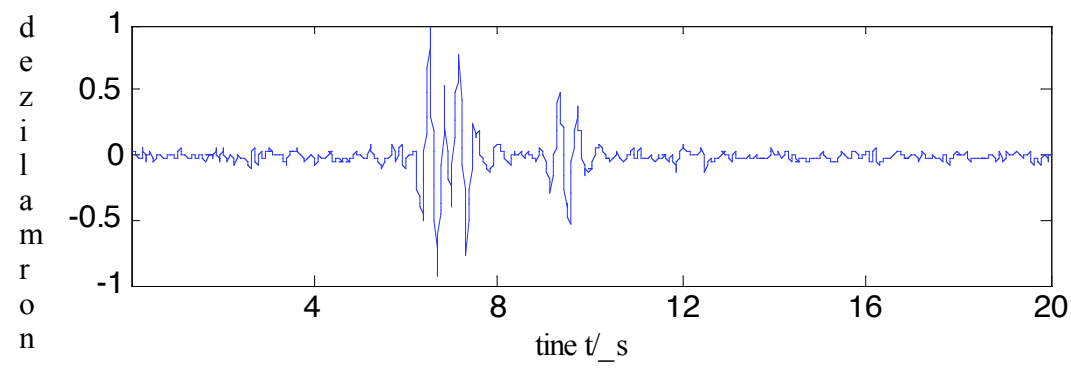

Fig. (7). A-scanning signal at position $y_{k}-y_{k}^{\prime}$.

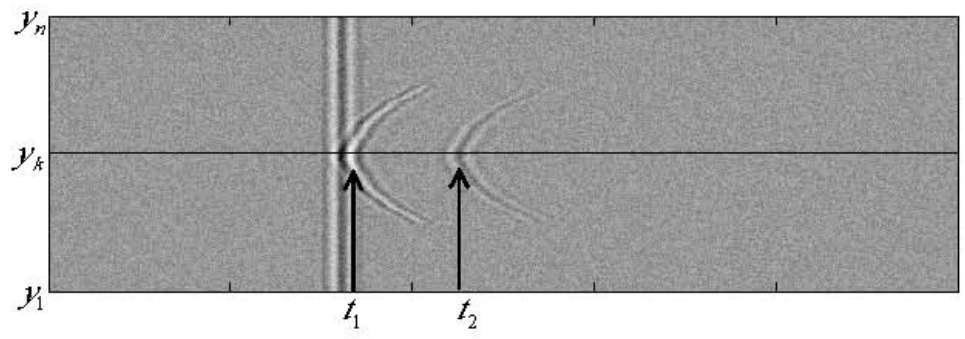

Fig. (8). B-scanning image after straightening and reconstructing processes.

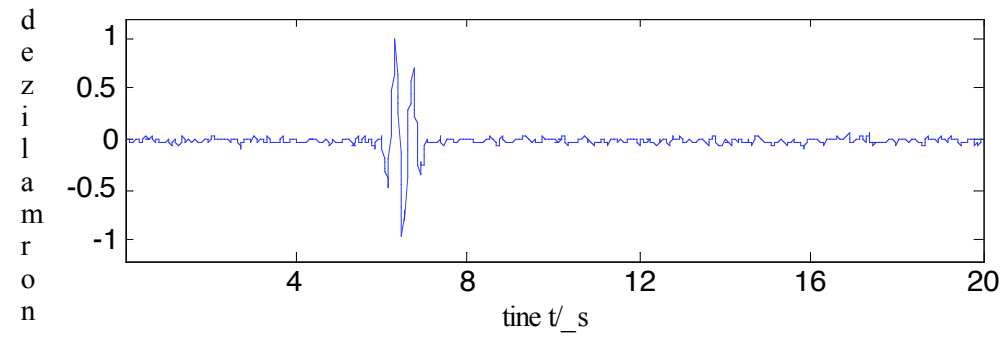

Fig. (9). Reference direct wave signals.

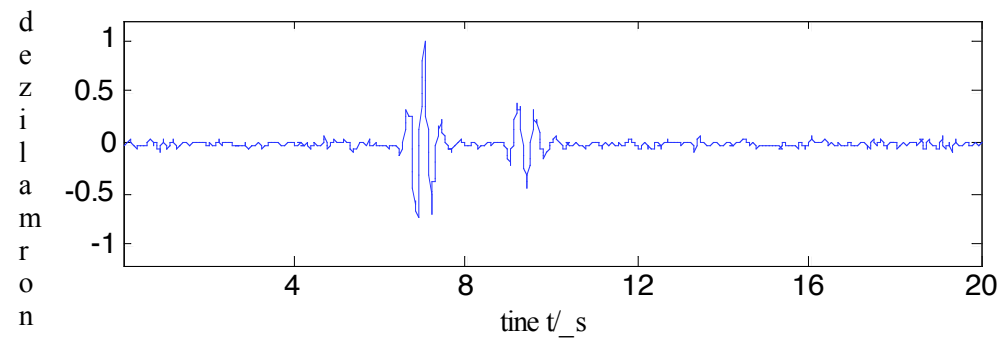

Fig. (10). A-scanning signal after filtering direct waves.

The reference through wave signals can be obtained by using energy distribution method as shown in Fig. (9). For the B-scanning image shown in Fig. (8), through waves in every A-scanning signal are filtered, so that the A-scanning signal in Fig. (7) undergoes shifting and through waves filtering to produce a signal as shown in Fig. (10). Fig. (11) shows the synthesized new B-scanning image after digital processing. It can be seen that through waves in B-scanning 


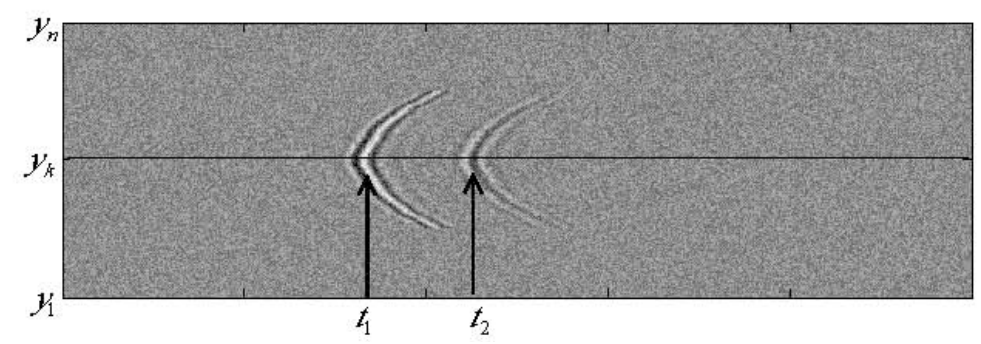

Fig. (11). B-scanning image after filtering direct waves.

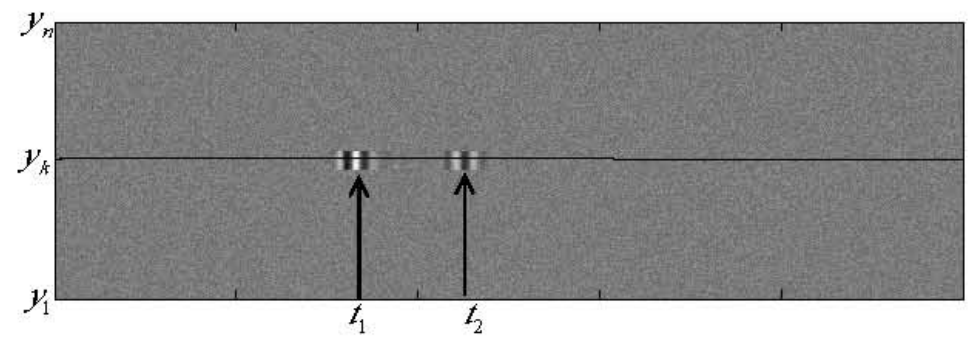

Fig. (12). B-scanning image after synthetic aperture focusing process.

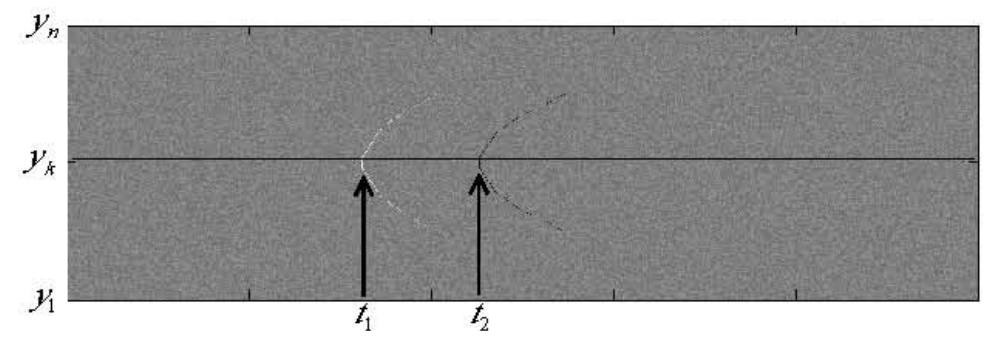

Fig. (13). B-scanning image after peak value capturing process.

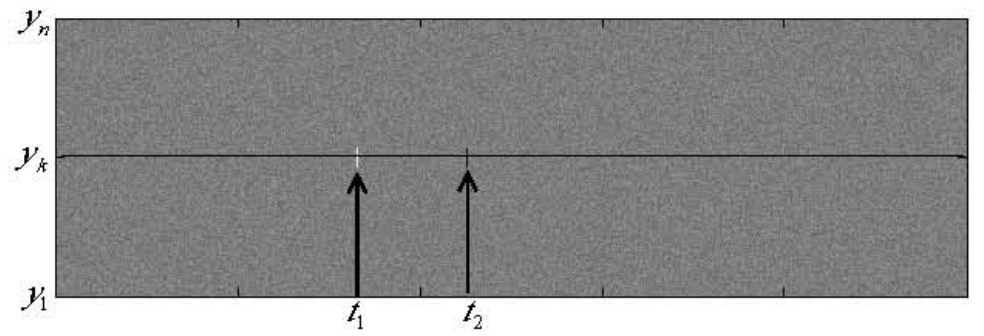

Fig. (14). B-scanning image after crack signal enhancement process.

image are completely filtered, and defect signals buried within through wave signals are fully displayed. Hence, this image processing achieves good results.

After the removal of through waves as shown in Fig. (11), the image then undergoes synthetic aperture focusing with window width of 13 , and the reconstructed image is shown in Fig. (12). This can effectively increase the horizontal resolution of the image. In addition, to effectively increase the vertical resolution, Fig. (11) also undergoes peak value capturing process according to the method mentioned in Fig. (5), the result is as shown in Fig. (13).

After peak value capturing process, the image further undergoes synthetic aperture focusing process and the reconstructed image is shown in Fig. (14). It can be observed that the characteristics of detected cracks can be more effectively captured in the processed image. Also, the vertical timing and horizontal positioning information of the crack in the image can be read faster and more accurately.

The arrival times of the diffraction signals on boundary points of the crack are captured in both original B-scanning image and the reconstructed image after the enhancement of defect signals. By applying equation (1) to calculate the defect depths from upper and lower boundary points, the size of the crack can be obtained. The measurement results are shown in case 1 of Table 1.

The same ultrasonic TOFD testing system is used to detect a welded steel plate of same size with a built-in vertical crack with length of $15 \mathrm{~mm}$, upper boundary point to surface distance of $13.5 \mathrm{~mm}$, and lower boundary point to surface distance of $28.5 \mathrm{~mm}$. The measurement results are shown in case 2 of Table 1 . 
Table 1. Measuring results of the crack size (mm).

\begin{tabular}{|c|c|c|c|c|}
\hline Case & \multicolumn{2}{|c|}{ 1 } & Original & Processed \\
\hline B-image & Original & Processed & 15.8 & 14.36 \\
\hline \hline Upper boundary point to surface distance & 1.12 & 1.52 & 29.37 & 13.57 \\
\hline Lower boundary point to surface distance & 17.5 & 16.23 & 15 & 14.54 \\
\hline Measuring size & 16.38 & 15 & $90.5 \%$ & 15 \\
\hline Actual size & 15 & $97.4 \%$ & $96.9 \%$ \\
\hline Accuracy & $90.8 \%$ & & 9 \\
\hline
\end{tabular}

From Table 1, results indicate that by using the enhancing method, the accuracy of the crack size measured from the B-scanning image can be as high as $97.4 \%$ and $96.9 \%$ in case 1 and 2 . This accuracy has increased by $6.6 \%$ and $6.4 \%$ respectively with regard to the original B-scanning image. Therefore, the method can achieve more accurate crack positioning and quantitative characterization.

\section{CONCLUSION}

This paper has proposed an enhancement method targeting ultrasonic TOFD for B-scanning image defect signals. A mathematical model has been constructed to solve the problem of identifying the defect in blind area on the near surface. Based on SAFT, this paper has proposed a method to improve the accuracy of localization and quantitative characterization of the crack. By analyzing the case study, this paper has verified the validity of the method. The main conclusions are as follows:

(1) The paper has studied the imaging synthetic method of ultrasonic TOFD. A test is designed by using B-scanning ultrasonic TOFD method on welded metal plate. Diffracted A-scanning signals at different locations are captured and digitized. The digitized A-scanning signals are then synthesized chronologically to form a B-scanning image in ultrasonic TOFD.

(2) Targeting at the problem of the inaccurate detection of defect within the blind area near surface by using ultrasonic TOFD, this paper has proposed a method to eliminate through waves by filtering, straightening and reconstructing. First, each A-scanning signal is adjusted based on the position where its amplitude reaches maximum. Then, the through waves are straightened. According to the energy distribution of B-scanning images, through wave signals are extracted to be used as reference signals. Finally, the reference signals are filtered out from every A-scanning signal to produce an output image, which is the B-scanning image after the through wave filtering. The results from the case study have shown that the defect on the blind area near surface can be extracted effectively.

(3) The image resolution for ultrasonic TOFD method is low, which causes inaccuracy in measuring results. To solve this problem, this paper first established a mathematics model according to the generation process of the B-scanning image. Based on the model, through waves are straightened and filtered according to the energy distribution law. Also, this paper has proposed an algorithm of synthesis aperture focusing based on the peak capturing pretreatment. The results from the case study have shown that the enhancing method for defect signals in the B-scanning image can improve the accuracy of crack localization and quantitative characterization.

\section{CONFLICT OF INTEREST}

The authors confirm that this article content has no conflict of interest.

\section{ACKNOWLEDGEMENTS}

This work described in the paper was supported by National Natural Science Foundation of China (51175401 and 51205230).

\section{REFERENCES}

[1] Organization of China Special Equipment Inspection Association, Ultrasonic Testing, China Labor and Social Security Publication Company: Beijing, 2007.

[2] L. Luo, "Diffraction method for ultrasonic testing of blind spots on the surface of different algorithms", Non-destructive Testing, vol. 36, pp. 45-46, May, 2012.

[3] V. Schmitz, S. Chakhlov, and W. Muller, "Experiences with synthetic aperture focusing technique in the field", Ultrasonic, vol. 37 , pp. 731-738, 2000.

[4] R. Zhang, and X. Wan, "Diffraction-echo ultrasonic transit-time method for quantitative nondestructive estimation of weld crack in situ", Chinese Journal of Mechanical Engineering, vol. 36, pp. 5457, 2000.

[5] D. Chi, T. Gang, and Y. Yao, "A near-surface defects detection based on ultrasonic TOFD method pattern", Welding Journal, vol. 32 , pp. 25-28, 2011.

[6] N. Ido, H. Hatanaka, and T. Arakawa, "Examination of flaw detection near the surface by the ultrasonic TOFD method", Key Engineering Materials, vol. 270-273, pp. 378-383, 2004.

[7] R. Miralles, L. Vergara, A. Salazar, J. Igual, "Blind detection of nonlinearities in multiple-echo ultrasonic signals", IEEE Transactions on Ultrasonic, Ferroelectrics, and Frequency Control, vol. 55, pp. 637-647, 2008.

[8] L. Wei, Z. Y. Huang, and Q. P. Wen, "Sparse deconvolution method for improving the time-resolution of ultrasonic NDE signals", NDT\&E International, vol. 42, pp. 430-434, 2009.

[9] M. Spies, and W. Jager, "Synthetic for defect reconstruction in anisotropic media", Ultrasonic, vol. 41, pp. 125-131, 2003.

[10] R. Sicard, J. Goyette, and D. Zellouf, "A SAFT algorithm for lamb wave imaging of isotropic plate-like structures", Ultrasonic, vol. 39, pp. 487-494, 2002.

[11] G. Yu, "Research on key Technologies of High Quality Ultrasound TOFD Imaging”, Doctoral Thesis, Zhejiang University, Hangzhou, China, 2013. 
[12] A. N. Sinclair, J. Fortin, B. Shakibi, F. Honarvar, M. Jastrzebski, M. D. C. Moles "Enhancement of ultrasonic images for sizing of defects by time-of-flight diffraction", NDT\&E International, vol. 43, pp. 258-264, 2010.

[13] D. Chi, and T. Gang, "Planar defects in signal and image feature and recognition of ultrasonic TOFD method", Welding Journal, vol. 26, pp. 1-4, 2005.
[14] D. Chi, "Research Based on Weld Defect Characterization of Ultrasonic TOFD Method", Doctoral Thesis, Harbin Institute of Technology, Harbin, China, 2007.

[15] X. Zhao, T. Gang, and Y. Yuan, "Thin steel plate ultrasonic signal analysis of spot-welded joints", Welding Journal, vol. 26, pp. 101105, Nov. 2005.

Received: September 16, 2014

Revised: December 23, 2014

Accepted: December 31, 2014

(C) Li et al.; Licensee Bentham Open.

This is an open access article licensed under the terms of the Creative Commons Attribution Non-Commercial License (http://creativecommons.org/licenses/by-nc/3.0/) which permits unrestricted, non-commercial use, distribution and reproduction in any medium, provided the work is properly cited. 